Chapter 21

\title{
Impact of Cancer Treatment on Reproductive Health and Options for Fertility Preservation
}

\author{
Kenny A. Rodriguez-Wallberg \\ Additional information is available at the end of the chapter \\ http://dx.doi.org/10.5772/55980
}

\section{Introduction}

\subsection{Cancer in patients of reproductive age}

Cancer is in general regarded as a disease of elderly people. Nevertheless, although age is the most significant risk factor for cancer in both sexes, young adults and children may also develop cancer diseases. Overall, men have a $45 \%$ risk of developing cancer at some time during their lives. For women, this risk is a bit lower, approximately $37 \%$ [1] and thus, in many cases, male and female cancer patients would be young and may not have been started building their families at the time of diagnosis. In people younger than 39 years, the risk of cancer is of about $1 / 72$ for men and $1 / 51$ for women. This risk increases with aging and between 40-59 years, 1/12 men and 1/11 women will develop cancer [1].

The majority of children, adolescents, and young adults diagnosed with cancer today will become long-term survivors. One primary concern of cancer survivors will be the ability to reproduce and have children. Detrimental effects on the reproductive system following cancer treatment have shown to negatively affect quality of life[2], [3]. Large studies of women and men with cancer have reported that the risk of infertility related to their treatments may be an important issue for those who have not yet started or completed their family size [2], [4].

\section{Cancer treatment modalities with impact in reproductive health}

Cancer surgery may have impact in fertility by removing reproductive organs or damaging structures needed for reproduction. Chemotherapy and radiotherapy have toxic effects on the gonads and may in certain cases induce ovarian and testicular failure, affecting thus all aspects 
of reproductive health, including pubertal development, impairment of hormone production and sexual function in adults. Effects of cancer biological therapy on gametes and reproductive organs are not yet established.

\section{Cancer surgery may cause infertility}

Surgery is currently the most effective treatment for cancer and eventually up to $100 \%$ of patients may be cured when complete removal of the tumor is achieved. Surgery may also be indicated for cancer prophylaxis, such as the case of premalignant disease of the cervix in female patients. In those very early stages of cervix cancer, the conization of a significant part of the cervix, may offer to patients a complete disease-free survival. However, in case of loop excisions, even if they are small, surgery of the cervix may induce subfertility by affecting the normal functioning of the cervix and its glandular secretion. Infertility induced by those interventions may be overcomed by using assisted reproductive technologies, such as treating the patient with intrauterine insemination or performing In Vitro Fertilization, IVF.

Surgery may also affect future fertility if there is removal or damage of the reproductive organs. In male patients, surgery for pelvic cancer such as for prostate, bladder or colon cancer may damage nerves and affect potency or ejaculation. Further on, surgical adjuvant treatment by removing the gonads may be indicated in female and male patients with hormone sensitive tumors.

In case of large tumors, neo-adjuvant chemotherapy and radiation may be indicated as first line treatment aiming to a reduction of tumor size and control of subclinical metastatic disease before surgical treatment. Neo-adjuvant therapy is usually planned before surgery in female patients with stage III breast cancer and young male cancer patients with bulky testicular cancer.

\subsection{Cancer surgery aiming at preserving fertility}

Fertility-sparing surgery may be an option for selected patients who wish to retain the ability to achieve a pregnancy. In many cases, pregnancies will occur spontaneously, nevertheless, causes of subfertility may be present in some patients, and a number of those may further require assisted reproduction treatments [5]. In gynecologic and urologic oncologic surgery, there has been a gradual development of fertility-sparing surgery aiming at preserving reproductive organs without compromising survival. Indications for fertility-sparing surgery include, in general, a well-differentiated low-grade tumor in its early stages or with low malignant potential.

\subsection{Fertility sparing surgery in female patients}

Table 1 presents a compilation of current data on fertility sparing surgery for young female patients with gynaecological cancer. In cases of selected ovarian tumors i.e. borderline tumors, 
young female patients may be offered a single oophorectomy aiming at preserving the uterus and the contralateral ovary[6].

$\begin{array}{ll}\text { Diagnosis } & \text { Type of surgery } \\ \text { Cervical cancer stage } & \text { Radical vaginal } \\ \text { IA1,1A2,1B1 } & \text { trachelectomy } \\ & \\ & \\ \text { Borderline ovarian } & \text { Unilateral } \\ \text { tumors FIGO stage I } & \text { oophorectomy }\end{array}$

Ovarian epithelial Unilateral cancer stage I, grade 1 oophorectomy

$\begin{array}{ll}\begin{array}{ll}\text { Malignant ovarian } \\ \text { germ cell tumors/sex } \\ \text { cord stromal tumors }\end{array} & \begin{array}{l}\text { Unilateral } \\ \text { oophorectomy }\end{array} \\ & \\ \text { Endometrial } & \text { Hormonal } \\ \text { adenocarcinoma } & \text { treatment with } \\ \text { Grade 1, stage 1A } & \text { progestational } \\ \text { (without myometrial } & \text { agents for 6 } \\ \text { or cervical invasion) } & \text { months }\end{array}$

\section{Description}

Laparoscopic pelvic

lymphadenectomy. Vaginal resection of the cervix and surrounding parametria keeping the corpus of the uterus and the ovaries intact

\section{Removal of the affected} ovary only, keeping in place the unaffected one and the uterus

\section{Removal of the affected} ovary only, keeping in place the unaffected one and the uterus

Removal of the affected ovary only

Follow-up with endometrial biopsies every 3 months

\section{Obstetric outcome Oncologic outcome}

Spontaneous

Rates of recurrence and

pregnancies

mortality are

described in up to comparable to those

$70 \%$. Risk of second described for similar trimester pregnancy cases treated by means loss and preterm of radical hysterectomy delivery or radiation therapy

\section{Pregnancies have}

Oncologic outcome is been reported and comparable with the favorable obstetric more radical approach outcome of removing both ovaries and the uterus. Recurrence $0-20 \%$ vs $12-58 \%$ when only cystectomy was performed Pregnancies have $7 \%$ recurrence of the been reported and ovarian malignancy and favorable obstetric 5\% deaths outcome

Pregnancies have Risk of recurrence been reported and similar to historical favorable obstetric controls outcome

Pregnancies have

Recurrence rate been reported 30-40\%. Five percent recurrence during progesterone treatment

Table 1. Fertility-sparing interventions in female patients. Reprinted, with permission from Rodriguez-Macias Wallberg et al, J Pediatric Blood \& Cancer, 2009, Ref [6].

The most established surgical procedure for fertility preservation of women is the radical trachelectomy described first by Dargent in 1994 [7]. It is currently offered in cases of invasive cervical cancer in early stages to patients interested in preserving fertility. About 500 cases have been reported worldwide, most of them in European countries, Japan, U.S.A, Canada and China [8-12]. 
The global utilization of fertility-sparing surgery in female patients is currently unknown. A recent European study collecting data from several countries demonstrated a low incidence of those procedures and it raised concerns on the need to centralize fertility sparing treatments of gynaecological cancer at accredited units, to ensure a sufficient number of patients at each center aiming at maintaining thus healthcare quality [13].

\subsection{Cervical cancer and fertility sparing surgery during pregnancy}

In pregnant women, the gynaecological cancer most commonly diagnosed is also the cancer of the cervix, usually detected at an early stage in those patients. The treatment of pregnant women should be established in the same way as in non-pregnant patients, based on the stage of the disease according to the International Federation of Gynecology (FIGO). Nevertheless, individualization of the treatment should be considered based on the desire to continue the pregnancy, the gestational age and the risks of modifying or delaying cancer treatment during the pregnancy. Clinical practice guidelines by the European Society for Medical Oncology ESMO are available on this respect [14]. Both abdominal radical trachelectomy [15] and vaginal trachelectomy [16] with lymphadenectomies have been reported during pregnancy to preserve an ongoing pregnancy and female fertility.

\subsection{Fertility sparing surgery in males}

In men, a partial orchidectomy has become an established method to preserve hormonal and sperm production in carefully selected patients. This method, originally developed for treatment of benign teratomas in prepubertal patients, has shown good results when adopted for treatment of testicular malignancies in adults [17]. Data from The German Testicular Cancer Study Group reported a 98.6\% disease-free survival rate at 7 years follow-up after conservative surgery of tumors $<2 \mathrm{~cm}[18]$.

\section{Radiotherapy treatments and their impact in reproductive health}

Radiation therapy is a component of curative therapy for a number of diseases, including those presenting frequently in young patients such as breast cancer, Hodgkin's disease, head and neck cancer and gynecologic cancers. It is often indicated for the treatment of prostate cancer as well.

It is known that cancer cells present with defects in their ability to repair sub-lethal DNA whereas normal cells have the ability to recover. Although radiation therapy is aimed to a locoregional application and although cancer cells are the target, radiation may also induce damage to normal cells in the tissues.

The response to radiation therapy depends on various factors such as the phase of cell cycle the cells are (cells in late G1 and S are more resistant), the degree of cell ability to repair the DNA damage and other factors such as hypoxia (hypoxic cells are more resistant), tumor mass and growth fraction. Non-dividing cells are more resistant than dividing cells. 
Except for the bone marrow, the most sensitive organs to radiation therapy in the body are the gonads, both the male testis and the female ovary. The extent of damage in the female and male gonads depends on the dose, fractionation schedule and irradiation field [19] [20]. Radiation therapy can be administered as teletherapy, which aims at treating a large volume of tissue. For small volumes of tissue, such as in the case of cervix cancer in the female, radiation therapy can be administered in encapsulated sources of radiation that can be implanted directly into or adjacent to tumor tissue.

Whenever female reproductive organs are involved in the irradiated field, i.e., the ovaries, the uterus and the vagina may be compromised and damaged by direct irradiation. Scattered radiation may also damage reproductive organs. In the female, radiation therapy results in dose-related damage of the gonads by the destruction of primordial follicles, which constitute the nonrenewable follicle pool. In women, the degree and persistence of the damage is also influenced by age at the time of exposure to radiotherapy and due to a reduced reserve of primordial follicles in older women, the number of follicles remaining may be also be reduced at older ages [21]. Table 2 presents a compilation of current knowledge on the impact of radiation doses and age at radiotherapy in male and female gonadal function [22]. In general, a dose of about 2 Gy applied to the gonadal area destroys up to $50 \%$ of the ovarian follicle reserve. In pediatric patients, failure in pubertal development may be the first sign of gonadal failure in both sexes. Total body irradiation (TBI) given in conjunction with myeloablative conditioning prior to bone marrow transplantation is one of the most toxic treatments for the gonads and it is highly related to gonadal failure in both sexes [23] [24].

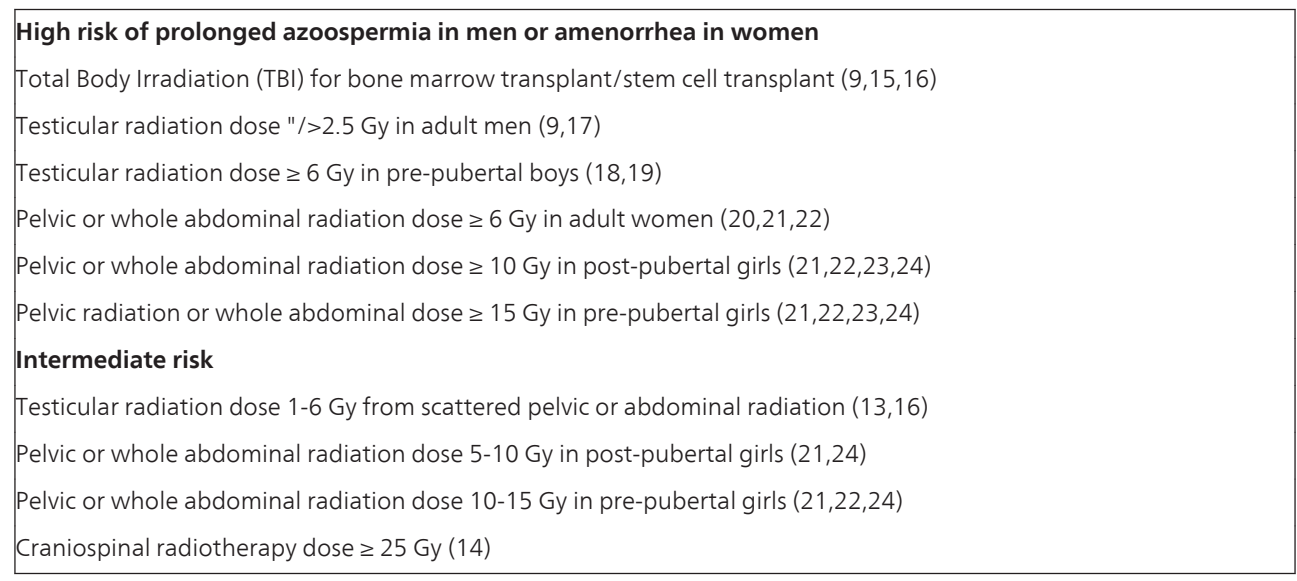

Table 2. Radiotherapy protocols with high or intermediate impact on ovarian and testicular function. Reprinted, with permission from Rodriguez-Wallberg and Oktay, J Ped Hematol Oncol, 2010, Ref [22].

In men, the gonadal stem cells responsible for the continual differentiation and production of mature spermatozoa, the spermatogoniae, are extremely sensitive to radiation. The Leydig cells, which are responsible for the hormonal production of testosterone, are on the contrary more resistant to radiotherapy and adult patients may thus preserve hormonal production 
although becoming infertile. In prepubertal boys, the sensitivity to radiation therapy of Leydig cells is greater than that of older males at very high doses [25]. Prepubertal patients may retain Leydig cell function after radiation therapy during childhood and in those cases they will present with normal pubertal development and well-preserved sexual function later in life. Nevertheless, most of those patients present at adulthood with reduced testicular size, impaired spermatogenesis and infertility.

\subsection{Gonadal shielding and ovarian transposition}

The standard medical procedure currently offered to reduce scatter radiation to reproductive organs and preserve fertility in male and female patients, both adult and prepubertal, is the use of shielding. When shielding of the gonadal area is not possible, the surgical fixation of the ovaries in females far from the radiation field known as oophoropexy (ovarian transposition) may be considered. It is estimated that this procedure significantly reduces the risk of ovarian failure by about $50 \%$ and those patients may retain some menstrual function and fertility [26]. Scattered radiation and damage of the blood vessels that supply the ovaries are related to the failure of this procedure [26].

\subsection{Radiotherapy of the uterus}

Radiotherapy of the uterus in young women and girls has shown to induce tissue fibrosis, restricted uterine capacity, restricted blood flow and impaired uterine growth during pregnancy, as shown by follow-up of cancer survivors [27] [28]. The uterine damage seems to be more pronounced in the youngest patients at the time of radiotherapy. As a consequence, radiotherapy-treated female patients present with a high risk of unfavorable pregnancy outcomes such as spontaneous abortion, premature labor and low birth weight offspring [27] [28]. Irradiation of the vagina is related to fertility and sexual issues due to loss of lubrication, anatomical impairments and in some cases vaginal stenosis.

\subsection{Cranial irradiation and hormonal dysfunction}

Cranial irradiation may induce disruption of the hypothalamic-pituitary-gonadal axis, which is a recognized potential complication that can lead to infertility in both female and male patients. Follow-up of female patients treated for brain tumors with cranial irradiation postand pre-pubertally has evidenced a high incidence of primary hypothalamic and pituitary dysfunction with consecuent disturbance in gonadotropin secretion. In some cases, precocious puberty may also be induced by cranial irradiation in childhood, which has been attributed to cortical disruption and loss of inhibition by the hypothalamus.

\section{Impact of chemotherapy in reproductive health}

Chemotherapy given as only treatment may be curative for a series of cancer presenting in young adults and children. In a vast majority of cancer treatments, chemotherapy proto- 
cols combine several agents and there is a possibility of a synergistic gonadotoxic effect[29]. In the female, primordial ovarian follicles including their oocytes and granulosa cells are particularly sensitive to alkylating agents, which induce apoptosis, as demonstrated in vitro [26], and in vivo using human ovarian tissue xenotransplanted in SCID mouse [30]. Ovarian failure is thus common after alkylating treatment [22].

Because of a high ovarian reserve with high numbers of follicles in young women, the risk of developing ovarian failure and permanent infertility after a cancer treatment is lower in younger than in older women [21]. Younger patients at the time of cancer treatment have thus a higher chance of recovering ovarian function following chemotherapy, nevertheless their fertility window might be reduced, and they should be recommended not to delay childbearing for too long [31].

\subsection{Clinical evaluation of ovarian reserve}

The development of amenorrhea should be considered unfavorable as it may be due to permanent gonadal failure. On the other hand, the presence of cycles should not be interpreted as proof of fertility. In the clinical setting, a gynecological examination including ultrasonography and estimation of antral follicle counts together with the determination of hormones such as follicle-stimulating hormone (FSH) and estradiol, inhibin and antimullerian hormone (AMH), may help the clinician in evaluating patient's remaining ovarian reserve after a cancer treatment and providing counseling on her chances to obtain a pregnancy.

\subsection{Conception following chemotherapy}

Due to toxicity of cancer treatments on growing oocytes, patients should be advised to avoid conception in the $6-12$ month period immediately following completion of chemotherapy treatment [32]. There is a high risk of teratogenesis during or immediately following chemotherapy, nevertheless DNA integrity has shown to return over time after a cancer treatment and thus no increase in childhood malignancies or genetic malformations have been shown in a large follow-up of more than 4000 children of cancer survivors [33].

\subsection{Chemotherapy in males}

In male patients, prepubertal status does not provide protection from gonadal damage and alkylating agents at high doses induce germ cell injury although Leydig cell function is commonly preserved [29]. Because most chemotherapy agents are given as part of a combination regimen, it has been difficult to quantify the gonadotoxicty of individual drugs.

Table 3 summarizes the gonadotoxic impact of chemotherapy agents on the female ovary and male testis. 


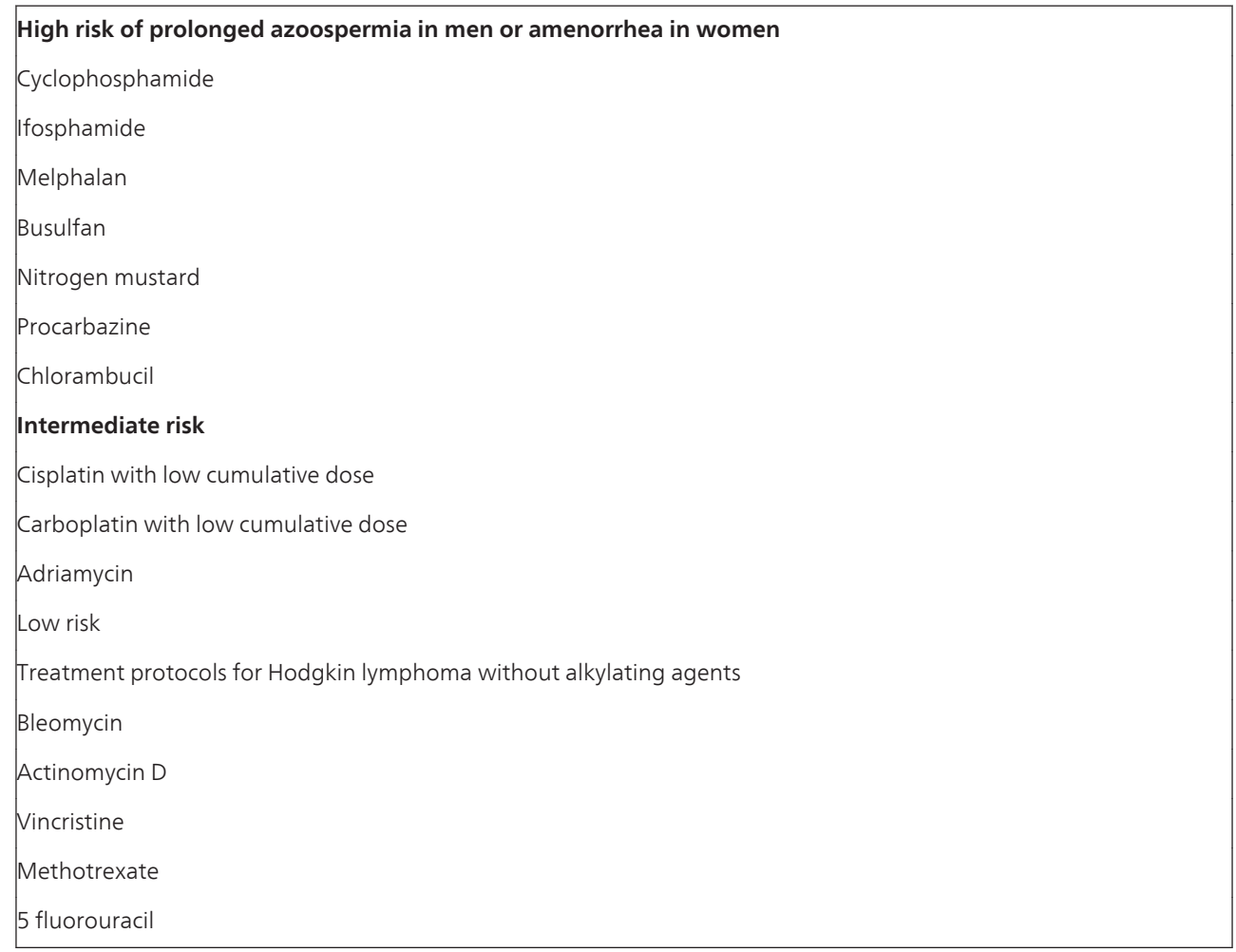

Table 3. Chemotherapy agents with high or intermediate gonadotoxic impact in women and men

\section{Options to preserve fertility by using cryopreservation methods}

In 2006, an expert panel commissioned by the American Society of Clinical Oncology ASCO published guidelines for fertility preservation for male and female patients [26]. Established cryopreservation methods for fertility preservation available for adult female and male cancer patients before starting cancer treatments included sperm freezing for male patients and embryo cryopreservation following ovarian stimulation with gonadotropins and In Vitro Fertilization, IVF for females. All remaining options were still considered experimental at that time and they included the freezing of unfertilized oocytes for adult women and the cryopreservation of gonadal tissue, ovarian or testicular, both methods still under development which constitute the only options that can be offered to pre-pubertal children (Figure 1).

Recently, by the end of 2012, the methods for cryopreservation of oocytes by vitrification techniques have markedly improved and thus freezing of unfertilized eggs is currently becoming an established clinical option for female patients. 


\section{Strategies for fertility preservation in males and females}

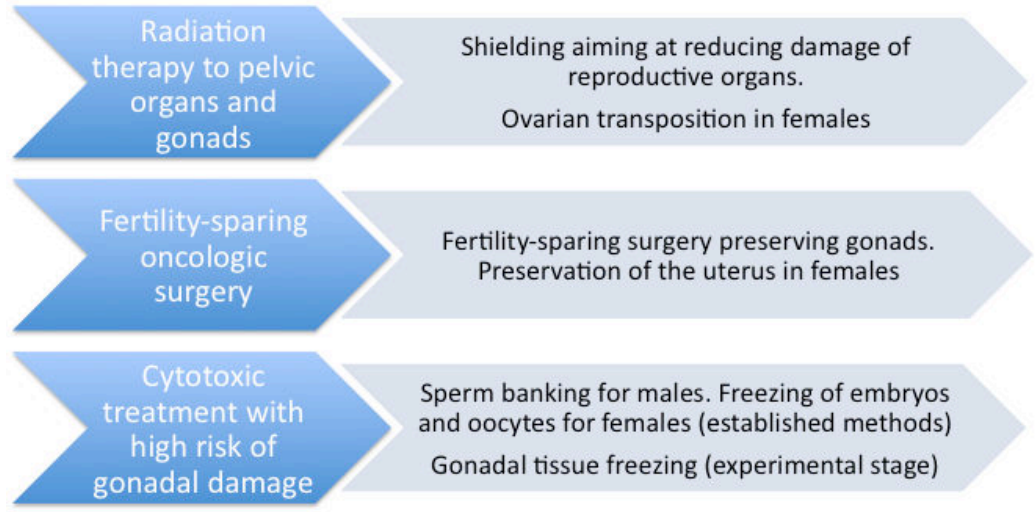

Rodriguez-Wallberg, 2013

Figure 1. Strategies for fertility preservation in males and females

\subsection{Sperm banking for male patients}

As many children are born after fertility treatments using frozen-thawed sperm, the cryopreservation of ejaculated semen is regarded as an established fertility preservation method in adult patients and pubertal boys. Although spermatogenesis starts in the pre-pubertal period and mature spermatozoa can be found at a Tanner III stage with a testis volume above $5 \mathrm{ml}$, spermatozoa production is generally effective only at the age of 13-14 years [33]. Sperm cryopreservation has been reported in adolescent patients from the age of 13 years with a high prevalence of normal sperm counts and semen volumes [34] [35]. Traditionally, sperm banking by cryopreservation of at least three semen samples with an abstinence period of at least 48 hours in between the samples has been recommended for adult males desiring to preserve their fertility [36].

In the situation of ejaculation failure, the search for spermatozoa in a urine sample could be proposed. When failure in obtaining a semen sample in young men and adolescents, a testicular sperm extraction TESE can be performed to retrieve spermatozoa [35]. Other methods described to retrieve spermatozoa in adolescents include penile vibratory stimulation and electro ejaculation.

\subsection{Cryopreservation of embryos or oocytes after controlled ovarian stimulation in females}

Adult women wishing to preserve fertility may undergo controlled ovulation stimulation with gonadotropins, for retrieval of matured oocytes and egg freezing, or, if the woman wishes, for in vitro fertilization (IVF) of the retrieved eggs and freezing of embryos. In general, controlled 
ovarian stimulation with gonadotropins for IVF may require only two weeks to achieve, as it has been shown that a random-start in the stimulation cycle, independently of cycle day, does not have a negative impact on the number and quality of oocytes retrieved.

Oocyte retrieval is undertaken usually by vaginal ultrasound assistance under sedation or general anaesthesia. Fertilization of the oocytes for embryo cryopreservation has traditionally been offered to woman having a partner. Transfer of frozen/thawed embryos today is a clinical routine in fertility clinics worldwide and it has been used for over 25 years. Intact embryos after thawing have similar implantation potential as fresh embryos and this treatment can lead to a $59 \%$ pregnancy rate and a $26 \%$ live birth rate [37].

Freezing unfertilized oocytes aiming at later thawing and fertilizing them by IVF is also a promising option to preserve fertility today. As the methods for cryopreservation of eggs have notably developed in recent years with the development of vitrification techniques, improving success in oocyte survival and fertilization rates has been achieved, approaching that of fresh oocytes. Worldwide, an increasing number of pregnancies and children born after fertilization offrozen-thawnoocyteshasbeenreportedandalthoughoverall pregnancy ratesarestillrelatively lower than those with embryo freezing [38-40], pregnancy rates and livebirths after thawing and fertilizing frozen eggs are currently reaching those obtained after embryo cryopreservation [41].

\subsection{Ovarian stimulation using aromatase inhibitors to maintain low systemic estradiol levels in case of breast cancer}

Ovarian stimulation with gonadotropins before egg retrieval aims at obtaining more than one oocyte per cycle and it is a key component of the success of IVF.

In women with an estrogen-sensitive tumor, the elevation of circulating estradiol levels during ovulation stimulation is undesirable and it has been regarded as potentially harmful. Therefore, hormone positive breast cancer patients have been largely excluded of the option to preserve fertility aiming at freezing eggs or embryos [42].

Alternative protocols, including natural cycle IVF (without hormone stimulation) or inducing ovulation by using Selective Estrogen Receptor Modulators (SERMs) and aromatase inhibitors alone or in combination with gonadotropins have been proposed, as they might be potentially safer. Natural cycle IVF gives only one oocyte or embryo per cycle and this treatment protocol has a high rate of cycle cancellation.

Both tamoxifen and letrozole can be administered alone or alongside with gonadotropins to increase the number of oocytes yielded for cryopreservation. Stimulation protocols using letrozole alongside with gonadotropins have shown to be most effective resulting in higher number of oocytes obtained and fertilized when compared to tamoxifen protocols [43]. The short-term follow-up of breast cancer patients having undergone ovarian stimulation with letrozole for fertility preservation has not shown any detrimental effects on survival [44].

Although aromatase inhibitors are contraindicated during pregnancy, data indicate that fertility treatments with letrozole are safe and the use of letrozole before conception does not induce any increased risks for the fetus [45]. Letrozole is currently used in the treatment of anovulatory infertility in many countries. 


\subsection{Cryopreservation of immature oocytes obtained without hormonal stimulation}

Freezing immature oocytes is also an option for female fertility preservation in case of patients having a contraindication for hormonal stimulation or when there is not time available for stimulation. The oocytes are retrieved in the natural cycle and frozen at an immature stage or after maturation in vitro (IVM) [46]. Immature oocytes survive cryopreservation better than mature metaphase II oocytes [47]. After thawing they can be matured in vitro and fertilized. In vitro maturation of oocytes is at an experimental stage and needs further development. Only few fertility centers worldwide offer treatments using this technique.

\subsection{Ovarian tissue freezing for prepubertal and adult patients}

As the vast majority of eggs making up the ovarian reserve are within primordial follicles in the ovarian cortex, small cortical ovarian biopsies may provide a high number of eggs to be preserved. This procedure is usually performed by laparoscopy, can be planned immediately after the diagnosis of malignant disease and does not require hormonal stimulation. In cases when the patient needs to undergo abdominal surgery for the treatment of cancer, the ovarian tissue can be harvested during the same surgical procedure. Although it is preferable to carry out cryopreservation of ovarian tissue before a gonadotoxic treatment, young women, adolescents and girls have normally an abundant number of primordial follicles in their ovaries and attempts to harvest ovarian tissue for cryopreservation may still be worthwhile after the first courses of chemotherapy, if the procedure was not possible before [6].

Cryopreservation of ovarian tissue is the only option in prepubertal girls, as sexual maturity is not required. As this procedure does not cause any significant delay to initiation of cancer treatment and it does not require ovarian stimulation, some adult female patients also prefer to preserve fertility by this method.

\subsection{Ovarian tissue transplantation}

Transplantation of frozen-thawn ovarian cortex has shown to be a new promising method for recovery of ovarian function [48] and in some cases sufficient to restore fertility [49-51]. Ovarian tissue can be transplanted orthotopically, i.e. at the anatomical intrapelvic ovarian site or heterotopically, i.e. at other places including extrapelvic sites $[52,53]$.

There have been hundreds of patients undergoing ovarian tissue freezing but only a small percentage of these have returned for ovarian transplantation.

Autotransplantation is only possible if absence of malignant cells in the graft is confirmed. Methods for detection of cancer cells in the ovarian tissue of patients having suffered from hematological malignancies are under development including immunohistochemistry or the polymerase chain reaction applied to the tissue [54]. The investigation of residual malignant cells in the ovarian tissue may also be performed by xeno-transplantation to immunodeficient SCID mouse. Autotransplantation of ovarian tissue in patients having suffered from systemic hematological malignancies is not recommended due to the high risk of retransmission of malignancy and only patients with cancer diagnosis associated with a negligible or no risk of 
ovarian compromise should be considered for future autotransplantation [55]. Ovarian tissue cryopreservation and transplantation has shown not to interfere with proper genomic imprinting in mice pups [56] but additional studies in other animal models are needed.

\subsection{In vitro culture of ovarian follicles}

Although many improvements have been reported on the in vitro culture of follicles at early stages aiming at developing them into competent mature follicles, those methods are still on development [57-59]. Follicles cultured isolated or within a piece of thawed tissue will be the option for patients with hematological and ovarian malignancies. The normality of imprinted genes of cryobanked oocytes cultured and matured in vitro has yet to be verified experimentally.

\subsection{Testicular tissue cryopreservation}

This technique involves removal of testicular tissue from the male patient before cytotoxic therapy is initiated. In prepubertal boys, as there is absence of spermatozoa and spermatids, studies have been going on to cryopreserve the testicular totipotent precursors, i.e. the spermatogonial stem cells. Success has been reported in cryopreservation methods of testicular tissue [60] but more research is still needed in how to use the frozen-thawed tissue and obtain mature spermatozoa in vitro. Research suggests that in vitro spermatogenesis is likely to be the safest option for boys suffering from haematological malignancies, which might be retransmitted by retransplantation, but this technique is still to be fully developed [61]. Although there are promising results in experimental animal studies of autologous retransplantation of spermatogonial stem cells showing re-colonization of seminiferous tubules generating complete spermatogenesis and mature germ cells and thus restoring natural fertility, the technique is still experimental in humans [61].

Cryopreservation of gonadal tissue offers hope to childhood cancer survivors, however it also raises several medical and ethical questions. Experimental methods for fertility preservation should only be offered to patients at specialized centers working with ethics board-approved research protocols and only in case when the recognized risks associated to the procedure are minimal.

\subsection{Ovarian suppression to prevent gonadal damage}

It has been hypothesized that suppressing the gonadal function transiently during chemotherapy could prevent ovarian follicle destruction in female patients by maintaining the follicles dormant. However, the pool of primordial follicles is normally non-proliferating. Those follicles lack FSH receptors [62] and their initial recruitment is not controlled by gonadotropins [63], therefore hormonal manipulation by suppressing gonadotropin release is not likely to affect them [64]. The vast majority of available studies having investigating gonadal protection by gonadotropin-releasing hormone analogues (GnRHa) agonists or antagonists during chemotherapy in females have been small, retrospective and uncontrolled. A significant number of those studies had used resumption of menses as a surrogate marker for fertility and many of them had reported higher frequency of resumption of menses in 
women having received GnRHa but none has demonstrated a beneficial effect regarding fertility recovery. Although data indicate that infertility is increased after a chemotherapy treatment, even if menstrual cycles are resumed [65], studies suggesting a beneficial effect of GnRHa co-treatment on preserving menstruation have had a great impact in the medical community and the empirical use of $\mathrm{GnRHa}$ for ovarian protection during chemotherapy is currently widely spread [26].

\section{Counseling and prompt referal increase the chances to preserve fertility}

Despite the fact that fertility issues are recognized in young people with cancer, health care professionals still report never referring cancer patients of reproductive age to a reproductive specialists for fertility preservation, indicating that many patients still do not receive adequate and timely information [66] [67]. This contrasts to data indicating that approximately three out of four cancer patients younger than 35 years and childless at the time of cancer treatment may be interested in having children in the future [2]. Because incidence of most cancers increases with age, the trend of delaying childbearing in Western societies will naturally result in more female cancer patients interested in fertility preservation.

In despite of this, recent data indicate that female cancer patients are still poorly informed on fertility threats of cancer treatment and options to preserve fertility in comparison with their gender counterparts. A recent Swedish survey found that less than half of female patients recalled having received appropriate information on reproductive threats of cancer therapy whereas $80 \%$ of male patients recalled having had an appropriate discussion [67]. Only a small number of female patients used fertility preservation methods compared to a rate $>70 \%$ of sperm freezing in male patients in that study. Urgency to start cancer treatment and lack of appropriate time, lack of knowledge on fertility preservation and awareness of the costs of assisted reproduction methods are recognized barriers to counseling and referring patients to fertility preservation [68].

\section{Conclusion}

Infertility due to gonadal failure is one of the major consequences of cancer therapy, particularly in patients who receive aggressive chemotherapy and/or radiotherapy treatment. Many surveys of cancer survivors have found that those patients are at increased risk of emotional distress if they become infertile as a result of their treatment. Evidence suggests that long-term survival after treatment for cancer during childhood is associated with increased risk of impaired quality of life and higher frequency of psychosocial problems often related to infertility issues. Adolescent cancer survivors have increased anxieties about body image and dating, and pediatric cancer survivors are less likely to marry than matched controls. Although cancer survivors can become parents by adoption or gamete donation, most would prefer to have biological parenthood and biologically related children. 
Oncologists should thus be prepared to discuss the negative impact of cancer therapy on reproductive potential with their patients in the same way as any other risks of cancer treatment are discussed. Furthermore, patients interested in fertility preservation should be promptly referred to a reproductive medicine expert to offer timely and appropriate counseling and improve success of fertility preservation. Close collaboration between the oncology team and the reproductive endocrinologists should be encouraged.

\section{Author details}

Kenny A. Rodriguez-Wallberg ${ }^{1,2,3}$

Address all correspondence to: kenny.rodriguez-wallberg@karolinska.se

1 Clinical Responsible of Fertility Preservation Programme, Karolinska University Hospital, Stockholm, Sweden

2 Karolinska Institutet, Department of Clinical Science, Intervention and Technology, Division of Obstetrics and Gynecology, Sweden

3 Karolinska University Hospital Huddinge, Fertility Unit, Stockholm, Sweden

\section{References}

[1] SEER Cancer Statistics Review 1975-2007, N.C.I., updated January 7, 20112011.

[2] Schover, L.R., et al., Having children after cancer. A pilot survey of survivors' attitudes and experiences. Cancer, 1999. 86(4): p. 697-709.

[3] Rosen, A., K.A. Rodriguez-Wallberg, and L. Rosenzweig, Psychosocial distress in young cancer survivors. Semin Oncol Nurs, 2009. 25(4): p. 268-77.

[4] Schover, L.R., et al., Knowledge and experience regarding cancer, infertility, and sperm banking in younger male survivors. Journal of clinical oncology : official journal of the American Society of Clinical Oncology, 2002. 20(7): p. 1880-9.

[5] Wong, I., et al., Assisted conception following radical trachelectomy. Human reproduction (Oxford, England), 2009. 24(4): p. 876-9.

[6] Wallberg, K.A., V. Keros, and O. Hovatta, Clinical aspects of fertility preservation in female patients. Pediatr Blood Cancer, 2009. 53(2): p. 254-60.

[7] Dargent, D., et al., La trachelectomie elargie(te), une alternative a l'hysterectomie radicale dans le traitement des cancer infiltrants developpes sur la face externe du col uterin. 1994. 2: p. 285-292. 
[8] Morice, P., et al., [Effects of radiotherapy (external and/or internal) and chemotherapy on female fertility]. Bull Acad Natl Med, 2010. 194(3): p. 481-92; discussion 492-4, 529-30.

[9] Liou, W.S., et al., Innovations in fertility preservation for patients with gynecologic cancers. Fertil Steril, 2005. 84(6): p. 1561-73.

[10] Abu-Rustum, N.R. and Y. Sonoda, Fertility-sparing surgery in early-stage cervical cancer: indications and applications. J Natl Compr Canc Netw, 2010. 8(12): p. 1435-8.

[11] Plante, M., et al., The vaginal radical trachelectomy: An update of a series of 125 cases and 106 pregnancies. Gynecologic oncology, 2011. 121(2): p. 290-7.

[12] Li, J., et al., Radical abdominal trachelectomy for cervical malignancies: Surgical, oncological and fertility outcomes in 62 patients. Gynecologic oncology, 2011. 121(3): p. 565-70.

[13] Kesic, V., et al., Fertility Preserving Management in Gynecologic Cancer Patients: The Need for Centralization. International Journal of Gynecological Cancer, 2010. 20(9): p. 1613-9.

[14] Pentheroudakis, G., et al., Cancer, fertility and pregnancy: ESMO Clinical Practice Guidelines for diagnosis, treatment and follow-up. Ann Oncol, 2010. 21 Suppl 5: p. v266-73.

[15] Ungar, L., et al., Abdominal radical trachelectomy during pregnancy to preserve pregnancy and fertility. Obstet Gynecol, 2006. 108(3 Pt 2): p. 811-4.

[16] Sioutas, A., et al., Three cases of vaginal radical trachelectomy during pregnancy. Gynecologic oncology, 2011. 121(2): p. 420-1.

[17] Sabanegh, E.S. and A.M. Ragheb, Male fertility after cancer. Urology, 2009. 73(2): p. 225-31.

[18] Heidenreich, A., et al., Organ sparing surgery for malignant germ cell tumor of the testis. The Journal of urology, 2001. 166(6): p. 2161-5.

[19] Gosden, R.G., et al., Impact of congenital or experimental hypogonadotrophism on the radiation sensitivity of the mouse ovary. Human reproduction (Oxford, England), 1997. 12(11): p. 2483-8.

[20] Speiser, B., P. Rubin, and G. Casarett, Aspermia following lower truncal irradiation in Hodgkin's disease. Cancer, 1973. 32(3): p. 692-8.

[21] Wallace, W.H.B., R.A. Anderson, and D.S. Irvine, Fertility preservation for young patients with cancer: who is at risk and what can be offered? The lancet oncology, 2005. 6(4): p. 209-18.

[22] Rodriguez-Wallberg, K.A. and K. Oktay, Fertility preservation medicine: options for young adults and children with cancer. Journal of pediatric hematology/oncology : official journal of the American Society of Pediatric Hematology/Oncology, 2010. 32(5): p. 390-6. 
[23] Sklar, C., Growth and endocrine disturbances after bone marrow transplantation in childhood. Acta paediatrica (Oslo, Norway : 1992) Supplement, 1995. 411: p. 57-61; discussion 62 .

[24] Thibaud, E., et al., Ovarian function after bone marrow transplantation during childhood. Bone marrow transplantation, 1998. 21(3): p. 287-90.

[25] Shalet, S.M., et al., Vulnerability of the human Leydig cell to radiation damage is dependent upon age. The Journal of endocrinology, 1989. 120(1): p. 161-5.

[26] Lee, S.J., et al., American Society of Clinical Oncology recommendations on fertility preservation in cancer patients. Journal of clinical oncology : official journal of the American Society of Clinical Oncology, 2006. 24(18): p. 2917-31.

[27] Wo, J.Y. and A.N. Viswanathan, Impact of radiotherapy on fertility, pregnancy, and neonatal outcomes in female cancer patients. International journal of radiation oncology, biology, physics, 2009. 73(5): p. 1304-12.

[28] Green, D.M., et al., Ovarian failure and reproductive outcomes after childhood cancer treatment: results from the Childhood Cancer Survivor Study. Journal of clinical oncology : official journal of the American Society of Clinical Oncology, 2009. 27(14): p. 2374-81.

[29] Hudson, M.M., Reproductive outcomes for survivors of childhood cancer. Obstetrics and gynecology, 2010. 116(5): p. 1171-83.

[30] Oktem, O. and K. Oktay, A novel ovarian xenografting model to characterize the impact of chemotherapy agents on human primordial follicle reserve. Cancer research, 2007. 67(21): p. 10159-62.

[31] Wallberg, K.A.R.-M., V. Keros, and O. Hovatta, Clinical aspects of fertility preservation in female patients. Pediatric blood \& cancer, 2009. 53(2): p. 254-60.

[32] Meirow, D., et al., Administration of cyclophosphamide at different stages of follicular maturation in mice: effects on reproductive performance and fetal malformations. Human reproduction (Oxford, England), 2001. 16(4): p. 632-7.

[33] Guerin, J.F., [Testicular tissue cryoconservation for prepubertal boy: indications and feasibility]. Gynecol Obstet Fertil, 2005. 33(10): p. 804-8.

[34] Bahadur, G., et al., Semen quality and cryopreservation in adolescent cancer patients. Hum Reprod, 2002. 17(12): p. 3157-61.

[35] Menon, S., et al., Fertility preservation in adolescent males: experience over 22 years at Rouen University Hospital. Hum Reprod, 2009. 24(1): p. 37-44.

[36] Meseguer, M., et al., Sperm cryopreservation in oncological patients: a 14-year follow-up study. Fertil Steril, 2006. 85(3): p. 640-5. 
[37] Marrs, R.P., J. Greene, and B.A. Stone, Potential factors affecting embryo survival and clinical outcome with cryopreserved pronuclear human embryos. Am J Obstet Gynecol, 2004. 190(6): p. 1766-71; discussion 1771-2.

[38] Oktay, K., A.P. Cil, and H. Bang, Efficiency of oocyte cryopreservation: a meta-analysis. Fertility and sterility, 2006. 86(1): p. 70-80.

[39] Practice Committee of the American Society for Reproductive Medicine, P.C.o.t.S.f.A.R.T., Ovarian tissue and oocyte cryopreservation. Fertil Steril, 2008. 90: p. 134-5.

[40] Medicine, P.C.o.t.A.S.f.R., ASRM Practice Committee response to Rybak and Lieman: elective self-donation of oocytes. Fertil Steril, 2009. 92(5): p. 1513-14.

[41] Noyes, N., E. Porcu, and A. Borini, Over 900 oocyte cryopreservation babies born with no apparent increase in congenital anomalies. Reprod Biomed Online, 2009. 18(6): p. 769-76.

[42] Rodriguez-Wallberg, K.A. and K. Oktay, Fertility preservation in women with breast cancer. Clinical obstetrics and gynecology, 2010. 53(4): p. 753-62.

[43] Oktay, K., et al., Fertility preservation in breast cancer patients: a prospective controlled comparison of ovarian stimulation with tamoxifen and letrozole for embryo cryopreservation. Journal of clinical oncology : official journal of the American Society of Clinical Oncology, 2005. 23(19): p. 4347-53.

[44] Azim, A.A., M. Costantini-Ferrando, and K. Oktay, Safety of fertility preservation by ovarian stimulation with letrozole and gonadotropins in patients with breast cancer: a prospective controlled study. Journal of clinical oncology : official journal of the American Society of Clinical Oncology, 2008. 26(16): p. 2630-5.

[45] Tulandi, T., et al., Congenital malformations among 911 newborns conceived after infertility treatment with letrozole or clomiphene citrate. Fertil Steril, 2006. 85(6): p. 1761-5.

[46] Chian, R.C., et al., Live birth after vitrification of in vitro matured human oocytes. Fertil Steril, 2009. 91(2): p. 372-6.

[47] Boiso, I., et al., A confocal microscopy analysis of the spindle and chromosome configurations of human oocytes cryopreserved at the germinal vesicle and metaphase II stage. Hum Reprod, 2002. 17(7): p. 1885-91.

[48] Oktay, K. and G. Karlikaya, Ovarian function after transplantation of frozen, banked autologous ovarian tissue. The New England journal of medicine, 2000. 342(25): p. 1919.

[49] Andersen, C.Y., et al., Two successful pregnancies following autotransplantation of frozen/ thawed ovarian tissue. Hum Reprod, 2008. 23(10): p. 2266-72.

[50] von Wolff, M., et al., Cryopreservation and autotransplantation of human ovarian tissue prior to cytotoxic therapy--a technique in its infancy but already successful in fertility preservation. European journal of cancer (Oxford, England : 1990), 2009. 45(9): p. 1547-53. 
[51] Oktay, K., I. Turkcuoglu, and K.A. Rodriguez-Wallberg, Four spontaneous pregnancies and three live births following subcutaneous transplantation of frozen banked ovarian tissue: what is the explanation? Fertility and sterility, 2011. 95(2): p. 804 e7-10.

[52] Oktay, K., et al., Embryo development after heterotopic transplantation of cryopreserved ovarian tissue. Lancet, 2004. 363(9412): p. 837-40.

[53] Sonmezer, M. and K. Oktay, Orthotopic and heterotopic ovarian tissue transplantation. Best Pract Res Clin Obstet Gynaecol, 2010. 24(1): p. 113-26.

[54] Meirow, D., et al., Searching for evidence of disease and malignant cell contamination in ovarian tissue stored from hematologic cancer patients. Hum Reprod, 2008. 23(5): p. 1007-13.

[55] Ajala, T., et al., Fertility preservation for cancer patients: a review. Obstet Gynecol Int, 2010. 2010: p. 160386.

[56] al, S.e., Immature cryopreserved ovary restores puberty and fertility in mice without alteration of epigenetic marks. PLoS One, 2008.

[57] Telfer, E.E., et al., A two-step serum-free culture system supports development of human oocytes from primordial follicles in the presence of activin. Hum Reprod, 2008. 23(5): p. 1151-8.

[58] Smitz, J., et al., Current achievements and future research directions in ovarian tissue culture, in vitro follicle development and transplantation: implications for fertility preservation. Hum Reprod Update, 2010. 16(4): p. 395-414.

[59] Telfer, E.E. and M. McLaughlin, In vitro development of ovarian follicles. Semin Reprod Med, 2011. 29(1): p. 15-23.

[60] Keros, V., et al., Methods of cryopreservation of testicular tissue with viable spermatogonia in pre-pubertal boys undergoing gonadotoxic cancer treatment. Hum Reprod, 2007. 22(5): p. 1384-95.

[61] Jahnukainen, K., et al., Intratesticular transplantation of testicular cells from leukemic rats causes transmission of leukemia. Cancer Res, 2001. 61(2): p. 706-10.

[62] Rice, S., et al., Stage-specific expression of androgen receptor, follicle-stimulating hormone receptor, and anti-Mullerian hormone type II receptor in single, isolated, human preantral follicles: relevance to polycystic ovaries. J Clin Endocrinol Metab, 2007. 92(3): p. 1034-40.

[63] McGee, E.A. and A.J. Hsueh, Initial and cyclic recruitment of ovarian follicles. Endocr Rev, 2000. 21(2): p. 200-14.

[64] Oktay, K., D. Briggs, and R.G. Gosden, Ontogeny of follicle-stimulating hormone receptor gene expression in isolated human ovarian follicles. The Journal of clinical endocrinology and metabolism, 1997. 82(11): p. 3748-51. 
[65] Letourneau, J.M., et al., The prevalence of self-reported reproductive impairment in young female cancer survivors throuhgout California. Fertil Steril, 2010. 94(4): p. 510 (Abstract).

[66] Forman, E.J., C.K. Anders, and M.A. Behera, Pilot survey of oncologists regarding treatment-related infertility and fertility preservation in female cancer patients. J Reprod Med, 2009. 54(4): p. 203-7.

[67] Armuand, G.M., Rodriguez-Wallberg K.A. et al., Sex differences in fertility-related information received by young adult cancer survivors. J Clin Oncol, 2012. 30(17): p. 2147-53.

[68] Quinn, G.P., et al., Patient-physician communication barriers regarding fertility preservation among newly diagnosed cancer patients. Soc Sci Med, 2008. 66(3): p. 784-9. 
